\title{
Functional characterization and identification of mouse Rad5 Id splice variants
}

\author{
Aaron M Gruver², Brian D Yard ${ }^{1}$, Campbell McInnes ${ }^{1}$, \\ Changanamkandath Rajesh ${ }^{1}$ and Douglas L Pittman*1
}

\author{
Address: ${ }^{1}$ Department of Pharmaceutical and Biomedical Sciences, South Carolina College of Pharmacy, University of South Carolina Campus, \\ Columbia, SC 29208, USA and 2Pathology and Laboratory Medicine Institute, Cleveland Clinic, Cleveland, OH 44195, USA \\ Email: Aaron M Gruver - gruvera@ccf.org; Brian D Yard - yard@cop.sc.edu; Campbell McInnes - mcinnes@cop.sc.edu; \\ Changanamkandath Rajesh - rajesh@cop.sc.edu; Douglas L Pittman* - pittman@cop.sc.edu \\ * Corresponding author
}

Published: 27 March 2009

BMC Molecular Biology 2009, 10:27 doi:10.1 186/1471-2199-10-27

This article is available from: http://www.biomedcentral.com/I47I-2/99//0/27

(C) 2009 Gruver et al; licensee BioMed Central Ltd.

This is an Open Access article distributed under the terms of the Creative Commons Attribution License (http://creativecommons.org/licenses/by/2.0), which permits unrestricted use, distribution, and reproduction in any medium, provided the original work is properly cited.
Received: 3I December 2008

Accepted: 27 March 2009

\begin{abstract}
Background: The homologous recombination (HR) pathway is vital for maintaining genomic integrity through the restoration of double-stranded breaks and interstrand crosslinks. The RAD5 I paralogs (RAD5IB, RAD5IC, RAD5ID, XRCC2, XRCC3) are essential for this process in vertebrates, and the RAD5ID paralog is unique in that it participates in both HR repair and telomere maintenance. RAD5ID is also known to directly interact with the RAD5IC and XRCC2 proteins. Rad5 Id splice variants have been reported in mouse and human tissues, supportive of a role for alternative splicing in HR regulation. The present study evaluated the interaction of the Rad5 Id splice isoform products with RAD5IC and XRCC2 and their expression patterns.
\end{abstract}

Results: Yeast-2-hybrid analysis was used to determine that the Mus musculus Rad5/d splice variant product RAD5ID $\Delta 7 \mathrm{~b}$ (deleted for residues 219 through 223) was capable of interacting with both RAD5IC and XRCC2 and that RAD5ID+int3 interacted with XRCC2. In addition, the linker region (residues 54 through 77) of RAD5ID was identified as a region that potentially mediates binding with XRCC2. Cellular localization, detected by EGFP fusion proteins, demonstrated that each of the splice variant products tested was distributed throughout the cell similar to the full-length protein. However, none of the splice variants were capable of restoring resistance of Rad5 I d-deficient cell lines to mitomycin C. RT-PCR expression analysis revealed that Rad5 Id 3 (deleted for exon 3) and Rad5 Id 5 (deleted for exon 5)transcripts display tissue specific expression patterns with Rad5 Id 3 being detected in each tissue except ovary and Rad5 Id 5 not detected in mammary gland and testis. These expression studies also led to the identification of two additional Rad5 Id ubiquitously expressed transcripts, one deleted for both exon 9 and 10 and one deleted for only exon 10.

Conclusion: These results suggest Rad5/d alternative splice variants potentially modulate mechanisms of HR by sequestering either RAD5IC or XRCC2. 


\section{Background}

Homologous recombination (HR) is responsible for repairing damage affecting both DNA strands and maintaining chromosome stability $[1,2]$. In mammals, HR requires the RAD51 family of proteins including RAD51 and the RAD51 paralogs (RAD51B, RAD51C, RAD51D, XRCC2, XRCC3) [3]. Genetic studies have demonstrated that RAD51 family members have non-redundant functions, as individual disruption of each gene confers increased sensitivity to DNA damaging agents and a genome instability phenotype [4-8]. In addition, the paralog proteins interact to form at least two stable complexes: a dimer consisting of RAD51C-XRCC 3 and a larger "BCDX2" complex consisting of RAD51B, RAD51C, RAD51D, and XRCC2 $[9,10]$. RAD51D is unique among the RAD51 family in that it is the only paralog currently known to support telomere maintenance in addition to the DNA repair functions [11].

Alternative pre-mRNA splicing is a mechanism responsible for proteome diversity and gene regulation in higher eukaryotes [12-14]. Splice variants of the Rad51d gene have been reported previously in mouse and human tissues, as well as in cancer derived cell lines [15-17]. Similarly, Rad51d alternative splice variants have also been identified in Arabidopsis [18]. For Mus musculus, seven alternative transcripts were identified that are predicted to encode six distinct putative protein isoforms. Alternatively spliced translation products commonly display different or antagonistic biological functions compared to their full-length counterparts [19]. Therefore, changes in the pattern of alternative splicing of regulatory genes could have an impact on physiology and pathogenesis, particularly tumor development and progression [20]. Splice variants of DNA repair genes potentially have the capability to regulate HR. It has been demonstrated that two splice variants of RAD52 increase the frequency of direct-repeat recombination from the same chromatid when expressed in either mammalian cells or yeast $[21,22]$. Moreover, mutations in the BRCA1 and BRCA2 genes, known to predispose carriers to breast and ovarian cancers, were found to disrupt exonic splicing enhancers and result in aberrant RNA splicing [23]. Recently, a RAD51 splice variant was uncovered that demonstrated homologous pairing activity similar to that of the full length RAD51 protein [24]. Here, we report the Mus musculus Rad51d alternative transcripts encode predicted proteins capable of making specific interactions with RAD51C and XRCC2 and the identification of two novel, ubiquitously expressed Mus musculus Rad51d alternative transcripts.

\section{Results}

\section{Alternative transcripts of Rad5 Id}

Multiple Rad51d transcripts were first detected by Northern blot analysis [25], and seven splice variants were later identified by RT-PCR in both mouse and human brain tissues [15]. The Rad51d gene consists of 10 exons, and a summary of the current evidence for each alternative transcript for the human and mouse Rad51d gene from the ASD and EASED databases is presented in Table $1[17,26]$. The Mus musculus Rad51d alternative transcripts are summarized in Figure 1A and for clarity are referred to as RAD51DA (exon excluded) or RAD51D+(intron included). The highly conserved ATP binding Walker Motifs A and B, present in all members of the RAD51 fam-

Table I: Alternative transcripts of the RAD5ID gene

\begin{tabular}{|c|c|c|}
\hline Variant Name & Representative EST/mRNA & Expression \\
\hline HsRAD5ID 3 & $\begin{array}{l}\text { AB016223, DA63092I, DA558919, DN997215, BX443779, } \\
\text { Bl823883, AL597240, NMI33627 }\end{array}$ & $\begin{array}{l}\text { Brain, kidney, chondrocytes, mammary gland (cancerous), } \\
\text { T Cell (Jurkat), lung, testis }\end{array}$ \\
\hline HsRAD5 ID 3,5 & AB0I6224, DA493777, NM_I33630 & Brain \\
\hline HsRAD5ID 3,4,5 & $\begin{array}{l}\text { AB016225, CD38786I, DA422600, DA8625I9, DBI32257, } \\
\text { DR005326, N57184, NMI33629 }\end{array}$ & Brain, trophoblasts, cervix, placenta, thymus, prostate \\
\hline HsRAD5ID 5 & $A B 018360$ & Brain \\
\hline HsRAD5 ID 4,5 & $A B 018361$ & Brain \\
\hline HsRAD5 ID+int3 4,5 & $A B 018362$ & Brain \\
\hline HsRAD5 ID+int3 & AB018363, B1915277, DN999128, DC391646 & Brain, bone marrow, spleen \\
\hline
\end{tabular}

\begin{tabular}{lll}
\hline MmRAD5ID 8 & AB052828, BB864057 & \\
MmRAD5ID 7b & AB052829 & Brain, bone marrow \\
MmRAD5ID 7,8 & AB052830 & Brain \\
MmRAD5ID 3 & AB05283I, BB629106 & Brain \\
MmRAD5ID 3,7b & AB052832 & Brain \\
MmRAD5ID 5 & AB052833 & Brain \\
MmRAD5ID+int3 & AB052834 & Brain \\
\hline
\end{tabular}



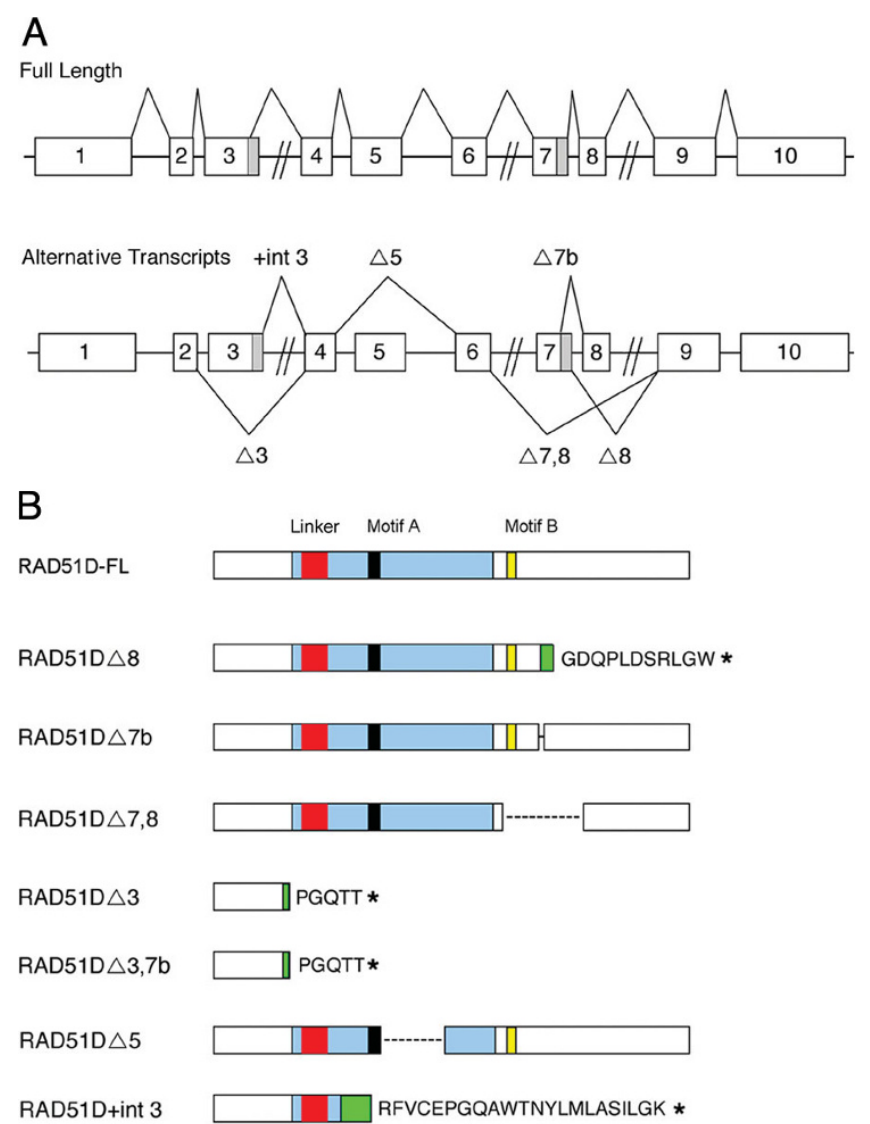

\begin{abstract}
Figure I
Summary of alternatively spliced transcripts of Mus musculus Rad5 Id. (A) The ten exons of Rad5 Id are shown as numbered boxes drawn relative to base pair length with both the full-length transcript (upper panel) and each alternatively spliced transcript shown (lower panel). Shaded areas designate partial intron retention (+int3) or partial exon exclusion $(\Delta 7 \mathrm{~b})$. (B) Predicted translation products of the RAD5ID isoforms are illustrated. Black and yellow boxes indicate the location of Walker Motifs $A$ and $B$ respectively. A core helix-hairpin-helix-GP rich domain (blue) and linker region (red) are also indicated. Amino acids introduced by splicing induced frameshift in the transcripts of RAD5ID $\Delta 8$, RAD5ID $\Delta 3$, and RAD5ID $\Delta 3,7 \mathrm{~b}$ are colored green with the corresponding novel sequence following. Asterisks represent sites of premature termination codons.
\end{abstract}

ily, are contained within exons 4 and 7 of Rad51d respectively (Figure 1B). RAD51D full length (FL) includes both exons $7 \mathrm{a}$ and $7 \mathrm{~b}$ in contrast to the RAD51D $\Delta 7 \mathrm{~b}$ alternative transcript in which the final 15 base pairs of exon 7 are excluded. Previously, this 3 ' portion of exon 7 as well as the retained intron in RAD51D+int3 were labeled as additional exons [15]. Internal deletions are also predicted in RAD51D $\Delta 7,8$ and RAD51D $\Delta 5$ (residues 193-
246, and 116-159 respectively), while stretches of novel amino acid sequence and premature stop codons are predicted for the RAD51D $\Delta 8$, RAD51D $\Delta 3$ and RAD51D+int3 isoforms as a result of splicing induced frameshift mutations (residues 224-233, 49-53, and 88-109 respectively). The splice variants RAD51D $\Delta 3$ and $\mathrm{RAD} 51 \mathrm{D} \Delta 3,7 \mathrm{~b}$ are predicted to encode identical peptides.

\section{RAD5 ID isoforms interact with members of the BCDX2 complex}

To investigate whether the predicted RAD51D isoforms interact with binding partners of the full-length protein, each was examined for interaction with RAD51C and XRCC2 by yeast two-hybrid analyses [27]. Yeast expressing the mouse RAD51D-RAD51C and RAD51D-XRCC2 binding partners display growth on selective medium indicating strong protein interactions. Replica plating results suggest that RAD51C interacts with RAD51D $\Delta 7 \mathrm{~b}$ while XRCC2 interacts with RAD51D $\Delta 8$, RAD51D $\Delta 7 \mathrm{~b}$ and RAD51D+int3 (Figure 2A). The activating domain (AD) fusion of RAD51C suggested interaction with RAD51D 43 . Mouse RAD51C-RAD51C displayed positive growth when tested in both orientations of the GAL4 fusion, which was not reported with the human RAD51C protein $[27,28]$.

Interaction between RAD51C, XRCC2 and the alternatively spliced isoforms of RAD51D was further assessed by measuring the activity of $\beta$-galactosidase. Colony lift assays were performed as a qualitative indicator of enzyme activity. As illustrated in Figure 2B, each of the matings that displayed growth on quadruple dropout medium were positive, with the exception of RAD51CRAD51C. $\beta$-galactosidase activity was weakest for the interactions of RAD51D $\Delta 8$ and RAD51D $\Delta 3$, suggesting a weaker or transient interaction with RAD51C and XRCC2. To quantify the degree of binding between RAD51D isoforms and RAD51C/XRCC2, $\beta$-galactosidase was measured using $o$-nitrophenyl- $\beta$-D-galactopyranoside as a substrate (Figure 2C). RAD51D $\Delta 7 \mathrm{~b}$ displays a level of interaction with RAD51C nearly identical to that of fulllength RAD51D. In agreement with colony lift assays however, binding between RAD51D $\Delta 3$ and RAD51C was suggested in only one orientation. RAD51D $\Delta 7 \mathrm{~b}$ and RAD51D+int3 associated with XRCC2 with an affinity similar to the full-length protein. The RAD51D $\Delta 8$, RAD 51D $\Delta 7,8$, and RAD 51D $\Delta 3$ isoforms display an ability to interact with XRCC2 $(36 \%, 29 \%$, and $22 \%$ the level of full-length RAD51D respectively), but variation in these interactions is observed depending upon the orientation of the GAL4 fusion.

\section{Domain mapping of RAD5 ID}

The observation that RAD51D isoforms may selectively interact with RAD51C and XRCC2 allowed further 
A

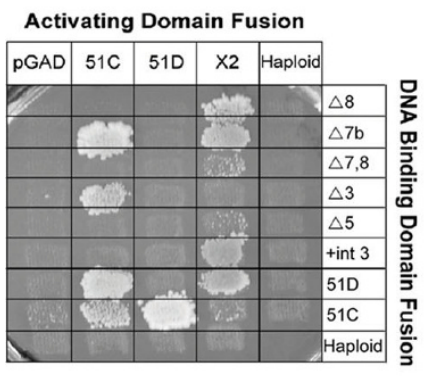

B

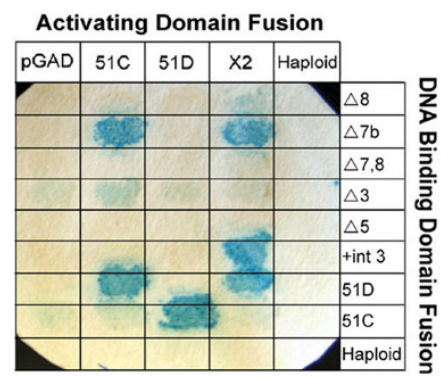

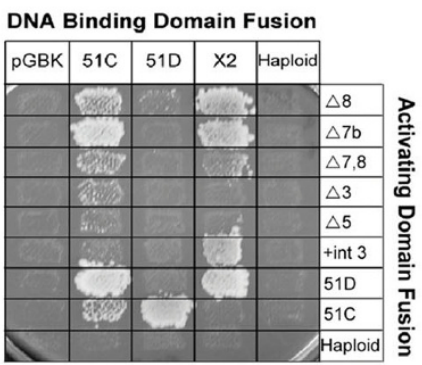

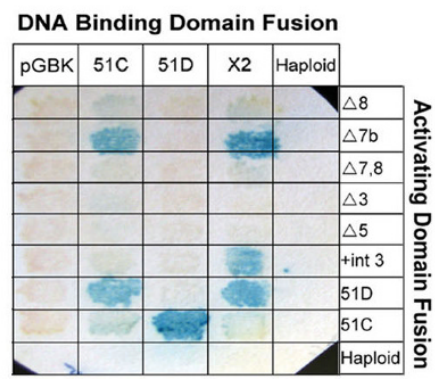

C

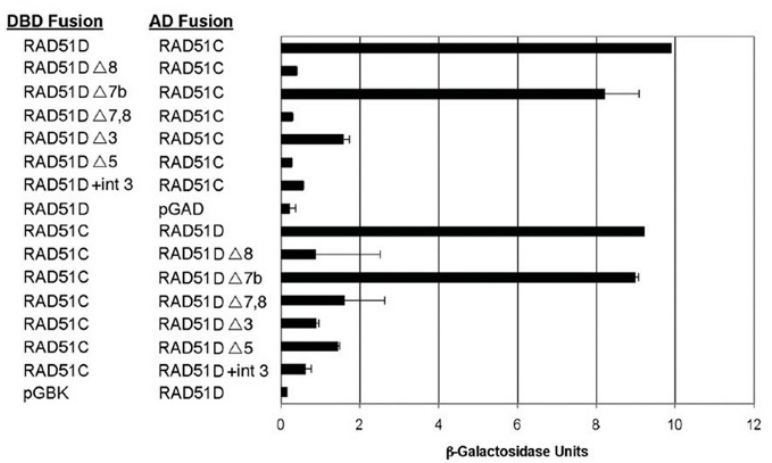

$\frac{\text { DBD Fusion }}{\text { RAD51D }}$
RAD51D $\triangle 8$
RAD51D $\triangle 75$
RAD51D $\triangle 7,8$
RAD51D $\triangle 3$
RAD51D $\triangle 5$
RAD51D+int
RAD51D
XRCC2
XRCC2
XRCC2
XRCC2
XRCC2
XRCC2
XRCC2
PGBK

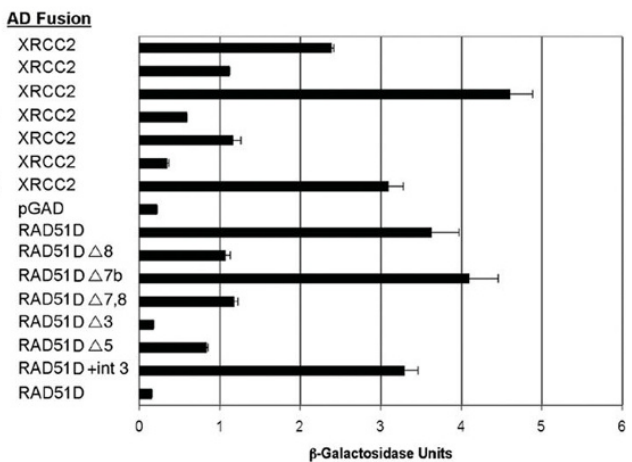

Figure 2

Interaction of the RAD5 ID alternative isoforms with RAD5 IC and XRCC2. (A) Sixty-four yeast two-hybrid interactions were tested by plating diploid strains on selective growth medium lacking adenine, leucine, histidine, and tryptophan. (B) Colony lift assays were performed to qualitatively assess $\beta$-galactosidase activity in mated yeast. $(C)$ Interactions between isoforms of RAD5 ID with RAD5IC and XRCC2 were quantified by measuring $\beta$-galactosidase activity using o-nitrophenyl- $\beta$-Dgalactopyranoside as a substrate. The binding capacity of the splice variants with RAD5 IC and XRCC2 are displayed. Experiments were performed in triplicate with error bars representing standard error of the mean. Abbreviations: 5 IC; RAD5IC, 5 ID; RAD5 ID-FL, X2; XRCC2, pGAD; pGADT7 vector, $p G B K$; PGBKT7 vector, $A D$; activating domain of GAL4, DBD; DNA binding domain of GAL4.

domain mapping of RAD51 paralog complexes. Miller et al. reported that the amino-terminal domain of RAD51D (residues 4-77) interacts with XRCC2, and the carboxyterminal region (residues 77-328) is sufficient for interaction with RAD51C [29]. Binding of RAD51D $\Delta 8$ (residues 4-233) and RAD51D+int3 (residues 4-109) to XRCC2 support this observation (Figure 2). However, the lack of association between RAD51D $\Delta 8$ and RAD51C suggests a more narrow region of the carboxy-terminal domain than previously reported, consisting of amino acids 234-329, is required for the interaction between RAD51D and RAD51C. Interestingly, the yeast two-hybrid analyses displayed in Figure 2 also suggest that residues 54-77 within the amino-terminal region determine whether RAD51D interacts with XRCC2 (shown in black, Figure 3C). To confirm these observations, the original RAD51D deletion constructs used in the study by Miller et al were tested against the RAD51D isoforms. If amino acids 54-77 are present, as with the RAD51D (4-77) construct, the peptide interacts with XRCC2 (Figure 3). When these residues are missing, as in the RAD51D $\Delta 3$ isoform, there is an absence of association with XRCC2 and some interaction with RAD51C. This span of 24 amino acids (54-77) is nearly identical to the "linker region" (residues 60-78) proposed from the structure modeling of human RAD51D from the Pyrococcus furiosus RAD51 crystal structure [29].

To further illustrate the position of the proposed region responsible for regulating these interactions, a protein homology model was constructed (DiscoveryStudio 2.0, Accelrys). RAD51D was matched to the RAD51 (RADA) structure from Pyrococcus furiosus (1PZN) and was shown to have $24 \%$ identity and $46 \%$ similarity. While the identity is somewhat low, the structural conservation of the RAD51 family supports that meaningful models can be obtained. After construction and refinement, the linker region is similar to what was proposed initially, but according to this model extends further into the alphahelices of the N-terminal domain (Figure 3E). The over- 
A

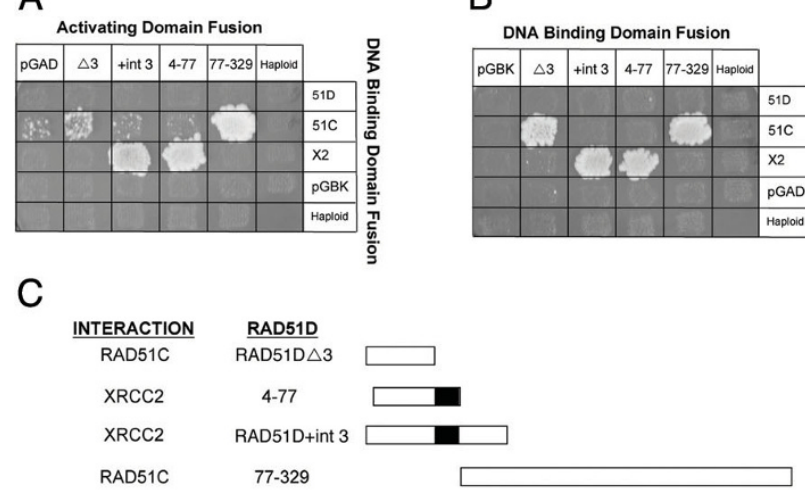

D

1 MGMLRAGLCP GLTEETVQLL RGRKIKTVAD LAAADLEEVA 41 QKCGLSYKAL VALRRVLLAQ FSAFPLNGAD LYEELKTSTA 81 ILSTGIGSLD KLLDAGLYTG EVTEIVGGPG SGKTQVCLCV 121 AANVAHSLQQ NVLYVDSNGG MTASRLLQLL QARTQDEEKQ 161 ASALQRIQVV RSFDIFRMLD MLQDLRGTIA QQEATSSGAV 201 KVVIVDSVTA VVAPLLGGOQ REGLALMMOL ARELKILARD 241 LGVAVVVTNH LTRDWDGRRF KPALGRSWSF VPSTRILLDV 281 TEGAGTLGSS QRTVCLTKSP RQPTGLQEMI DIGTLGTEEQ 321 SPELPGKQT

E
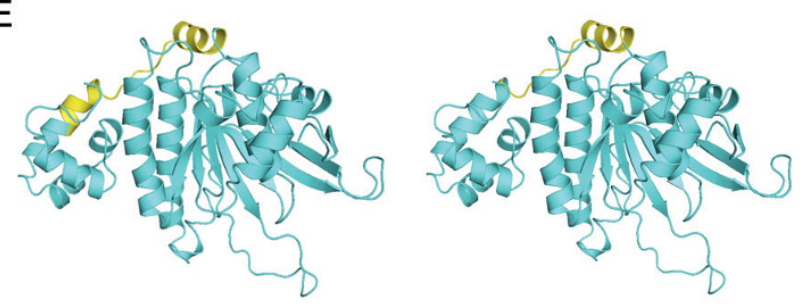

Figure 3

Domain mapping of Mus musculus RAD5 ID. (A-B)

Yeast two-hybrid analysis of RAD5ID $\Delta 3$ and RAD5ID+int3 are directly compared with the original deletion constructs of RAD5ID. (C) Diagram of the RAD5ID $\triangle 3$ and RAD5D+int 3 alternative splice constructs compared with the amino (4-77) and carboxy-terminal (77-329) regions of RAD5ID. The black box represents the predicted linker region of RAD5ID. (D) The region of the protein that allows interaction with XRCC2 is illustrated in bold type, while sequence required for its interaction with RAD5IC is presented in italics (note a small area of overlap between residues 223-233). The underlined 24 amino acid region (residues 54-77) appears to be critical for determining the specificity of the interaction between RAD5ID isoforms and XRCC2. (E) Homology model of RAD5 ID from the Pyrococcus furiosus RAD5 I crystal structure. The yellow highlighted region (left) represents the linker region and the yellow area (right) represents the 24 amino acid region proposed to determine XRCC2 specificity. Abbreviations: 5 ID; RAD5 IDFL, 5IC; RAD5 IC, X2; XRCC2, 4-77; residues 4-77 of the amino-terminal domain of RAD5ID, 77-329; residues 77329 of the carboxy-terminal domain of RAD5 ID, PGAD; pGADT7 vector, $p G B K$; $P G B K T 7$ vector. lapping region that potentially regulates RAD51D interaction with XRCC2 and RAD51C is located at the junction between the two domains previously demonstrated to interact with XRCC2 and RAD51C respectively.

\section{Localization of RAD5 ID isoforms}

RAD51D appears to be present throughout the cell although localization specific to telomeric regions in both meiotic and somatic cells has been demonstrated [11]. To determine if the multiple isoforms of RAD51D display similar localization, amino-terminal enhanced green fluorescent protein (EGFP) tagged constructs were generated and transiently expressed in Rad51d-deficient mouse embryonic fibroblasts (MEFs) (see Additional file 1, Panel A). Fluorescent microscopy reveals EGFP-RAD51D-FL is present in both the cytoplasm and nuclear compartments, similar to EGFP vector control. The RAD51D alternatively spliced isoforms also display a mixed cytoplasmic and nuclear distribution within the cell. In contrast, full length EGFP-RAD51C is predominantly nuclear in agreement with the presence of a non-canonical nuclear localization signal (NLS) in its carboxy-terminal region [30,31]. Fulllength EGFP-RAD51D was tested for the ability to repair mitomycin C (MMC) induced DNA damage in Rad51ddeficient MEFs. The percentage resistance was nearly identical to that observed from cells transfected with RAD51DFL (see Additional file, Panel B), suggesting the amino-terminal EGFP fusion results in a biologically active protein. Additionally, no change in the localization of EGFPRAD51D-FL was observed when expressed in wild-type MEFs. This confirms that failure to observe nuclear localization is not due to accumulated mutations in Rad51ddeficient MEFs (data not shown).

\section{Complementation analysis of RAD5ID isoforms}

Expression of RAD51D-FL was previously demonstrated to correct the DNA interstrand crosslink repair deficiency of Rad51d-deficientMEFs [6,32]. To determine whether RAD51D alternative splice products retain any ability to restore cellular resistance to DNA interstrand crosslinking damage caused by exposure to MMC, each was tested in a complementation assay [32]. In the present study, approximately $45 \%$ of the population expressing fulllength RAD51D was capable of resisting MMC challenge (Figure 4A). However, none of the alternative RAD51D isoforms restored resistance to DNA interstrand crosslinks when compared with vector control populations.

\section{Expression and identification of novel Rad5 Id alternative transcripts}

Mouse Rad51d alternative transcripts were originally identified in brain tissue [16]. However, the isolation of corresponding expressed sequence tags in a variety of tissues and cell types suggest that RAD51D isoforms are widely 


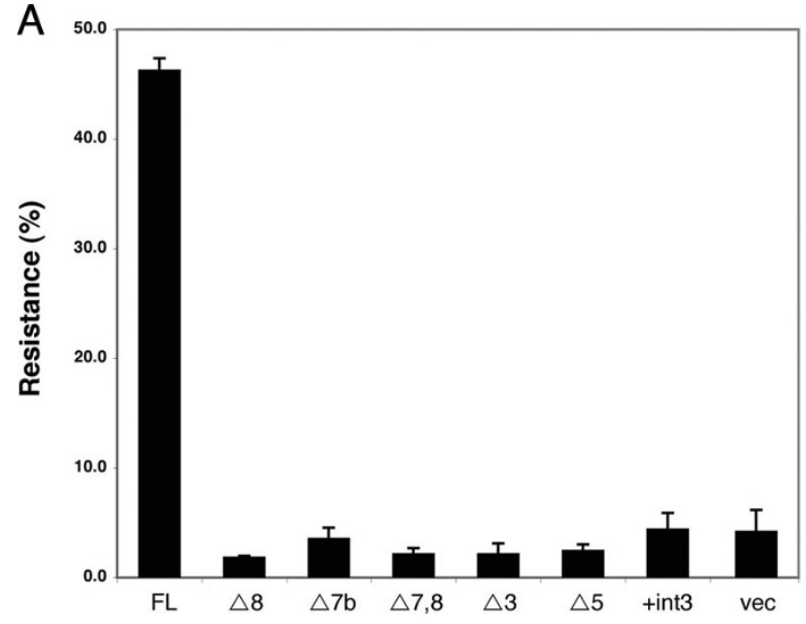

B

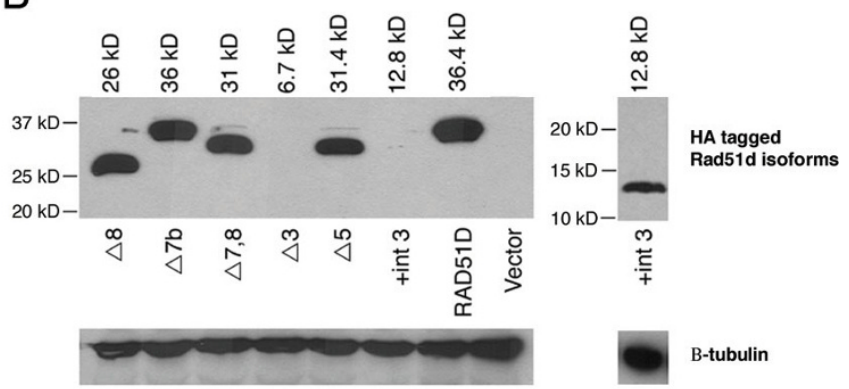

Figure 4

The putative translation products from alternative splicing of Mus musculus Rad5 Id fail to complement a Rad5 I d deletion. (A) Alternative transcripts of Rad5 Id

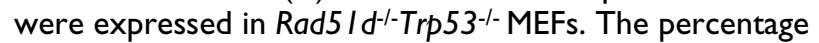
resistance indicates the fraction of transfectants that survived treatment with $4 \mathrm{ng} / \mathrm{mL}$ mitomycin C (MMC). Cells transfected with empty vector (vec) were included as controls. Error bars represent the standard error. (B) Western blot of

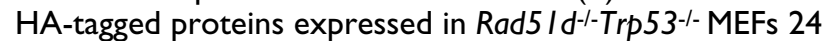
hr post-transfection (upper panel). The expected molecular mass of each isoform is shown above each. Immunoblotting for $\beta$-tubulin was performed as a loading control (lower panel). Expression of RAD5ID 33 could not be verified likely due to the relatively small molecular weight of the putative translation product $(6.7 \mathrm{kDa})$.

expressed (Table 1). To further investigate, RNA isolated from C57BL/6J mouse tissues were used for RT-PCR expression analysis. A series of overlapping PCRs were designed to detect all Rad51d alternative transcripts (Figure 5A). RAD51D $\Delta 3$ and RAD51D $\Delta 5$ were consistently detected in most tissues examined and their identities verified by restriction enzyme and sequence analysis (not shown). However, RAD51D $\Delta 3$ was not detected in ovarian tissue. Additionally, RAD51D $\Delta 5$ was not detected in mammary gland and testis, suggesting that these splice isoforms are differentially expressed. Because these same

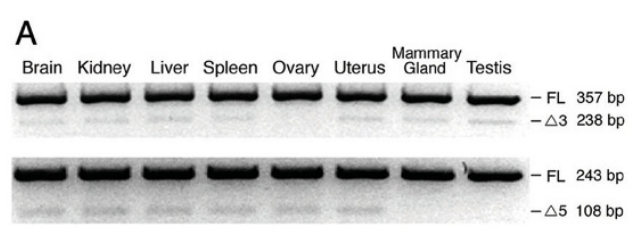

B

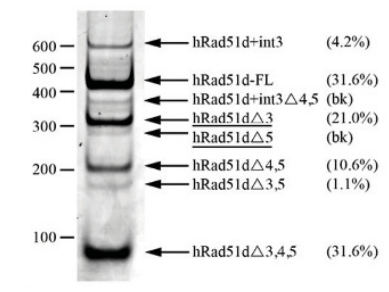

C

MRad51d 223: GLALMMOLARELKILARDLGVAVV VTNHLTRDWDGRRFKPAL mRad51d $\triangle 10$ 223: GLALMMQLARELKILARDLGVAVV VTNHLTRDWDGRRFKPAI mRad51d $\triangle 9,10$ 223: GLALMMQLARELKILARDLGVAVV LCLS*

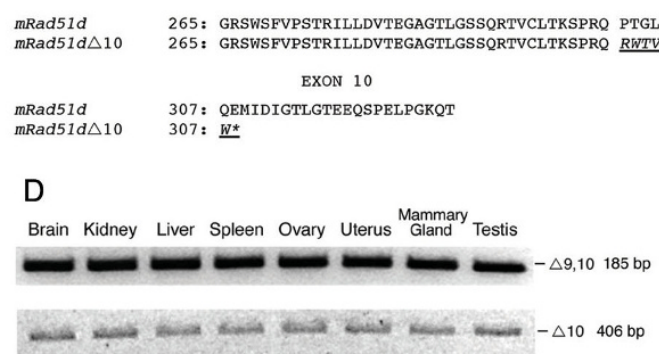

Figure 5

Expression analysis of Rad5 Id alternative transcripts. (A) RT-PCR for Mus musculus Rad5 I d and selected splice variants was conducted in eight tissues. Expression of RAD5ID $\Delta 3$ employing primers Rad5Id FI and RI (upper panel); RAD5ID 55 with Rad5 Id F2 and R2 (lower panel). (B) RT-PCR products of human RAD5 ID in normal breast. Arrows indicate transcripts corresponding to known human splice isoforms [16]. RAD5ID $\Delta 3$ and RAD5ID $\Delta 5$ transcript positions are underlined. Size markers in base pairs are shown on the left. Numbers in parenthesis represent transcript abundance as determined by ImageJ analysis of the band intensities (bk; background). (C) The RAD5ID peptide is aligned with the predicted amino acid sequence of RAD5 ID $\Delta 9,10$ and RAD5 ID $\Delta I 0$. RAD5 ID $\Delta 9,10$ contains the first 8 exons of RAD5ID followed by four out of frame amino acids (underlined and italicized) encoded by intron 8 . RAD5ID $\triangle 10$ includes amino acids I-302 of RAD5 ID followed by five novel amino acids encoded by intronic sequence 5980 bp downstream from the predicted Rad5 Id polyA site. Premature termination codons are indicated by an asterisk, and exon boundaries are represented by a gap in the alignment of the predicted amino acid sequences. (D) RT-PCR for RAD5ID $\Delta$ 9, I0 (upper panel) and RAD5ID $\Delta$ I0 (lower panel) from Mus musculus tissues with primers $\operatorname{Rad5}$ Id F3 and $\operatorname{Rad5Id} \Delta 9,10 \mathrm{RI}$ or $\operatorname{Rad} 5 \mathrm{Id} \Delta \mathrm{I} 0 \mathrm{RI}$ respectively. Abbreviations: 5 ID; RAD5 ID-FL, $\Delta 3$; RAD5ID $\Delta 3, \Delta 5$; RAD5ID $\Delta 5, \Delta 9,10 ;$ RAD5ID $\Delta 9,10, \Delta I 0 ;$ RAD5ID $\Delta I 0$. 
isoforms were identified in human adult and fetal brain cDNA libraries [16], initially named HsTRAD-d1 and HsTRAD-d4 respectively, expression analysis was performed in normal human breast tissue using primers that span from exon 2 to exon 6 . Consistent with the results in mouse tissue, $21 \%$ of the detectable products corresponded to RAD51D $\Delta 3$, whereas RAD51D $\Delta 5$ was not detectable above background levels (Figure 5B).

As part of our efforts toward verifying the presence of exons 7 through 10 in Rad51d splice variants [15], 3'RACE was performed on total RNA isolated from $\mathrm{BALB} / \mathrm{C}$ mouse liver tissue. Sequencing of cloned products revealed the existence of two additional alternatively spliced transcripts lacking sequence corresponding to one or both of the final Rad51d exons (Figure 5C). Both RAD51D $\Delta 9,10$ and RAD51D $\Delta 10$ transcripts are derived from the use of alternative splice sites contained within downstream intronic sequence and predicted to result in C-terminal truncated RAD51D isoforms. Notably for RAD51D $\Delta 10$, this alternative splice donor site is located 5980 bp downstream, past the predicted Rad51d polyadenylation site. In addition, both RAD51D $\Delta 9,10$ and RAD51D $\Delta 10$ predicted translation products contain a short stretch of novel sequence resulting from the ensuing frameshift. Using unique primers, both $\operatorname{RAD} 51 \mathrm{D} \Delta 9,10$ and RAD51D $\Delta 10$ were demonstrated to be expressed in all tissues examined (Figure 5D).

\section{Discussion}

The studies described here are the first to explore functional properties of RAD51D isoforms predicted to result from alternative splicing [15]. Each splice product was first tested for its ability to interact with RAD51C and XRCC2, proteins known to directly interact with RAD51D and participate as part of the BCDX2 complex [9]. BCDX2 is a complex that binds to DNA nicks, which is one of the key steps during homologous recombination repair of DNA crosslinks and branched structures, which are similar to Holliday junctions [9,33]. Additionally, BCDX2 performs strand-annealing reactions [33]. Previous domain mapping of RAD51 paralog interactions indicated that the amino-terminal portion of RAD51D interacts with XRCC2 and the carboxy-terminal region interacts with RAD51C [29].

The RAD51D+int3 isoform is predicted to contain residues 4-87 of the full-length protein and an additional 20 amino acids from the splice induced frameshift. Consistent with the Miller model [29], this isoform interacted strongly with XRCC2 and failed to interact with RAD51C (Figure 2). However, the RAD51D $\Delta 3$ isoform containing the N-terminal domain, but not the linker region, did not interact with XRCC2 but did interact with RAD51C, albeit dependent upon the orientation. These results suggested that residues $60-78$ within the RAD51D predicted linker region governs interactions between the XRCC2 and RAD51C paralogs. Therefore, within the CDX2 complex there may be three key interactions: 1) RAD51C with both the $\mathrm{N}$ and C-terminal RAD51D domains; 2) interactions of the RAD51D linker region with XRCC; and 3) interactions between the $\mathrm{N}$ and C-terminal RAD51D domains via a predicted salt bridge [34]. Electron microscopic analysis showed that RAD51D-XRCC2 alone formed a multimeric ring structure in the absence of DNA, similar to that of RAD52, but formed a filamentous structure unlike that of RAD51/RecA upon addition of ssDNA [35]. Regulating these three types of interactions would likely influence the formation and function of this complex during HR repair.

The RAD51D $\Delta 7 \mathrm{~b}$ isoform was the only alternative splice product to interact with both RAD51C and XRCC2. Presumably, internal deletions resulting from $\operatorname{RAD} 51 \mathrm{D} \Delta 7,8$ and RAD51D $\Delta 5$ affect integrity of the C-terminal domain, particularly the $\beta$-sheet [29]. RAD51D $\Delta 7 \mathrm{~b}$ is missing a small portion of exon 7, resulting in an in-frame deletion of "GQQRE" between residues 219-223. Because the deletion in RAD51D $\Delta 7 \mathrm{~b}$ is in proximity to the adenine nucleotide binding sequence of Walker Motif $\mathrm{B}$ in RAD51D [25,36], it is possibly required for the hydrolysis of ATP. The ATPase activity of RAD51D is required for its role in the repair of DNA interstrand crosslinks [32,37]. Residues adjacent to Motif $B$ have been found to be critical to ATPase activity in Walker box containing proteins including RAD51D. Notably, conserved glutamate residues in ATP-binding cassette proteins have been proposed to serve as a catalytic base in ATP hydrolysis [38]. Thus, the observation that RAD51D $\Delta 7 \mathrm{~b}$ did not complement sensitivity of Rad51d-deficient cells from DNA interstrand crosslinks caused by mitomycin $\mathrm{C}$ is consistent with a role for the "GGQRE" sequence in ATP hydrolysis.

The exclusion of an NLS has been reported or implied from studies of numerous alternative splice products. For example, predicted splice variants of both RAD51C and RAD51B are expected to lack their putative NLS sequences $[28,30,31,39]$. Given the telomere binding of endogenous RAD51D in human cells [11] and the observation that each of the predicted isoforms of RAD51D includes the "RKIK" putative NLS sequence at the amino terminus, nuclear localization of EGFP-RAD51D isoforms was expected. In contrast, RAD51D was expressed throughout the cell. Another published study, using EGFP-RAD51D, is consistent with this observation [31]. It is conceivable that the presence of the EGFP fusion in the amino-terminal region of RAD51D inhibits access to the "RKIK" sequence whereas the carboxy-terminal NLS present in RAD51C is unaffected. Alternatively, the over-expression of EGFP-RAD51D may saturate the cellular machinery 
responsible for import. Recently, a RAD51 alternative splice isoform was identified which also lacks exons 9 and 10 [24]. This hRAD51- $\Delta$ ex9 had subtle differences in tissue expression, being primarily expressed in testis. hRAD51- - ex9 was also able to perform strand invasion activity similar to full-length and was localized to the nucleus, likely through an RKR motif introduced by the translational frameshift. In either case, the likelihood remains that the "RKIK" sequence in RAD51D is not recognized as an NLS, and transport of RAD51D into the nucleus occurs as part of a protein complex [31].

The previous protein modeling and interaction studies suggested linker regions were critical for interaction of the RAD51 paralogs. Our results are consistent with this interpretation but also suggest this region may play a role in regulating these interactions. A small region of RAD51C was also identified to be critical for interaction with both RAD51B and XRCC3 [40]. It remains to be determined whether aberrant splicing of Rad51d and related HR genes play a role during carcinogenesis. However, two of the human alternative transcripts of RAD51D have been identified in tumor derived cells (Table 1) [17], including an EST from a mammary tumor (DN997215) that corresponds to the RAD51D $\Delta 3$ isoform. The association of alternative gene products in cancer raises the possibility that the alternative splicing mechanism is a potential target for gene therapy $[19,41,42]$. Molecular targeting of residues 54-77 in RAD51D could disrupt interactions within the BCDX2 recombinosome and sensitize cells to DNA interstrand crosslinks.

The pattern of protein-protein interactions between isoforms of RAD51D and other RAD51 paralogs described here suggests that RAD51D $\Delta 7 \mathrm{~b}, \mathrm{RAD} 51 \mathrm{D} \Delta 3$, and RAD51D+int3 have potential to regulate homologous recombination repair by sequestering members of BCDX2 complex. In addition, three of the alternatively spliced transcripts, RAD51D $\Delta 3$, RAD51D $\Delta 8$, and RAD51D+int3, harbor premature termination codons and are thus subject to degradation by nonsense-mediated mRNA decay (NMD) [43]. More than one third of all mRNAs derived from alternative splicing are estimated to contain similar nonsense codons [44]. For that reason, it has been proposed that the pre-mRNA splicing and NMD pathways are functionally coupled to provide an additional level of post-transcriptional regulation $[45,46]$. Therefore, cells could potentially alter gene expression by favoring the splicing of pre-mRNA into an alternative transcript that is targeted to NMD. It is also conceivable that HR may be directed on a tissue selective manner and that upregulation or downregulaton of specific isoforms provides a means of regulation. This hypothesis is supported by the observation that the Rad51d alternative transcripts display tissue specific expression patterns (Figure 5). It is also intriguing that RAD51D+int 3 corresponds to one of the alternative splice isoforms predicted in humans [16]. During the course of these studies, two new RAD51D isoforms were isolated, RAD51D $\Delta 10$ and $\operatorname{RAD} 51 \mathrm{D} \Delta 9,10$. Further studies are now necessary to explore the two newly discovered variants and determine whether splice isoforms may regulate HR. Finally, it remains to be determined whether these Rad51d alternatively spliced products interact with additional known RAD51D interacting proteins BLM and SWS1 $[47,48]$ or contribute to maintenance of telomere integrity.

\section{Conclusion}

Here we report the Mus musculus Rad51d alternative transcripts encode predicted proteins capable of making specific interactions with RAD51C and XRCC2. Expression studies revealed the RAD51D $\Delta 3$ and RAD51D $\Delta 5$ transcripts display tissue specific expression, being detected in each tissue except for mouse ovary or mammary gland and testis respectively. Additionally, we report the identification of two novel, ubiquitously expressed Mus musculus Rad51d alternative transcripts. The predicted RAD51D isoforms contain truncated C-terminal ends due to the retention of intron $8(\operatorname{RAD} 51 \mathrm{D} \Delta 9,10)$ and the deletion of exon $10(\operatorname{RAD} 51 \mathrm{D} \Delta 10)$. The unusual number of alternative splice variants expressed from the Rad51d gene compared with the other members of the RAD51 family suggests the RAD51D isoforms potentially regulate specific HR functions.

\section{Methods \\ Plasmid construction}

Complementary DNA clones encoding each predicted translation product from alternative splicing of Mus musculus Rad51d were generated [15]. The GenBank accession numbers are $\underline{\mathrm{AB} 052828.1}$ (RAD51D $\Delta 8$ ), $\underline{\mathrm{AB} 052829.1}$ (RAD51D $\Delta 7 \mathrm{~b}), \quad \underline{\mathrm{AB} 052830.1}$ (RAD51D $\Delta 7,8)$, $\underline{\mathrm{AB} 052831.1}$ AB052832.1 (RAD51DA3), $\underline{\mathrm{AB} 052833.1}$ (RAD51D 55$)$, and AB052834.1 (RAD51D+int3).

RAD51D $\Delta 8$ was generated by ligating a 734 bp sequence resulting from a NheI/StuI digest of full-length Rad51d to a dsDNA made by annealing $\operatorname{Rad} 51 \mathrm{~d} \Delta 8 \mathrm{StuI}$ and Rad51d $\Delta 8$ BamHI oligos. RAD51D $\Delta 7 \mathrm{~b}$ was produced by PCR based site directed mutagenesis using primers $\operatorname{Rad} 51 \mathrm{~d} \Delta 7 \mathrm{~b}$ F1and $\operatorname{Rad} 51 \mathrm{~d} \Delta 7 \mathrm{~b}$ R1. The RAD51D $\Delta 7,8$ expression construct was generated by multiple subcloning steps. First, full-length Rad51d was digested with XhoI/ BamHI, and the resulting 248 bp fragment cloned into pDsRed2-N1 (Clontech, Palo Alto, CA) to generate the RAD51D $\Delta 7,83^{\prime}$ end. Second, a 622 bp fragment resulting from NheI/AvaII digest of full-length Rad51d was isolated and subcloned into the NheI/AvaII sites of the 
RAD51D $\Delta 7,83$ ' end construct. Finally, duplexed oligos of $\operatorname{Rad} 51 \mathrm{~d} \Delta 7,8$ AvaII and Rad51d $\Delta 7,8$ Xhol were subcloned into the pDsRed2-N1 construct generated above prior to the final cloning step. RAD51D $\Delta 3$ was constructed by ligating the $200 \mathrm{bp}$ fragment resulting from a NheI/BstXI digest of full-length Rad51d to duplexed oligos Rad51d $\Delta 3$ BstXI. The 5' end of RAD51D $\Delta 5$ was amplified using Rad51dKpnIF and Rad51d $\Delta 5$ NheBamR and cloned into pUC19. The $3^{\prime}$ end was amplified using Rad51d $\Delta 5$ NheF and Rad51dBamR primers prior to subcloning into the RAD51D $\Delta 55^{\prime}$ pUC19 construct. RAD51D+int 3 was generated by a restriction digest of fulllength Rad51d using NheI and BsrFI enzymes. The resulting $323 \mathrm{bp}$ fragment was ligated with duplexed oligos Rad51d+int3F and Rad51d+int3R to construct the final insert. The make-up of each splice product cDNA was confirmed by double-strand sequencing and cloned into the NheI/BamHI sites of the pcDNA3.1/Hygro (+) vector (Invitrogen, Carlsbad, CA) with the exception of RAD51D $\Delta 5$, which was cloned into the KpnI/BamHI sites. Each construct contains an influenza HA epitope-tagging sequence at the 5 ' end.

Full-length Mus musculus Rad51c was obtained by reverse transcribing total RNA from mouse kidney as described above. The DNA was PCR amplified using Rad51cKpnF and Rad51cBamR primers. Full-length Mus musculus Xrcc2 was amplified from IMAGE clone 5357630 (American Type Culture Collection; Manassas, VA) using XRCC2F and XRCC2R primers. Rad51c and Xrcc2 amplification products were digested with $\mathrm{KpnI} / \mathrm{BamHI}$ and subcloned into the pcDNA3.1/Hyro(+) vector encoding an HA epitope tagging sequence at the 5' end. The RAD51D (477 ) and (77-329) deletion constructs were a generous gift of Dr. Joanna Albala (University of California, Davis, Sacramento, CA).

\section{Yeast two-hybrid analysis}

$M m R a d 51 d, M m R a d 51 c$, and MmXrcc2 inserts containing the $\mathrm{N}$-terminal hemaglutinin tag were cloned into the EcoRI/BamHI restriction sites of pGADT7 and pGBKT7 yeast two-hybrid vectors encoding the activating and DNA binding domains of GAL4 respectively (Clontech). Haploid transformants of AH109 and Y187 were generated using the EZ Transformation Kit II (Zymo, Orange, CA). Matings were performed on YPDA plates 16-24 hours prior to replica plating on dropout media. The presence of diploids was confirmed by growth on -Leu/-Trp medium following 3 days of incubation at $30^{\circ} \mathrm{C}$. Mated strains containing interacting proteins were subsequently analyzed for growth on -Ade/-Leu/-His/-Trp medium. Images were captured following 10 days of growth at $30^{\circ} \mathrm{C}$. Colony lift assays were performed according to the yeast protocols handbook (PT3024-1, Clontech). To determine the strength of protein-protein interactions, liquid $\beta$-galactos- idase assays were performed using ortho-nitrophenyl- $\beta$ galactopyranoside as a substrate (ONPG; Sigma, St. Louis, MO) essentially as described [15]. All $\beta$-galactosidase assays were performed in triplicate with constructs in both the GAL4 activating and DNA binding domains.

\section{Localization studies}

To generate green fluorescent protein fusions of the alternative splice variants, each was cloned into the KpnI/ BamHI sites of a pEGFP-C1 based vector (BD Biosciences). Constructs were transiently expressed in Rad51d-deficient MEFs grown on glass coverslips as described [6]. Cells were harvested 24 hours post-transfection and coverslips were washed in $1 \times$ PBS prior to fixation with $4 \%$ paraformaldehyde for 10 minutes at room temperature. Fixed cells were subsequently washed and permeablized with $0.3 \%$ Triton-X 100 for 5 minutes. Nuclei were counterstained with $0.2 \mu \mathrm{g} / \mathrm{mL}$ of 4 ',6-diamidino-2-phenylindole dihydrochloride hydrate (Sigma) in $1 \times$ PBS for 10 minutes and coded before mounting on glass slides with fluorescent mounting medium (DakoCytomation, Carpinteria, CA). Slides were viewed using a Nikon Eclipse fluorescent microscope, and images captured using a $60 \times$ dry lens objective.

\section{Complementation analysis}

Rad51d $\%$-Trp53\% MEFs were grown in monolayer culture as described [6]. One microgram of each plasmid construct was transfected into Rad51 $\mathrm{d}^{-}$Trp53\%- MEFs using Lipofectamine with Plus reagent in six-well format according to the manufacturer's instructions (Invitrogen). Twenty-four hours post-transfection cells were trypsinized, mixed, and divided equally $\left(\sim 7.5 \times 10^{5}\right.$ cells per dish) onto two $150 \mathrm{~mm}$ dishes. Twenty-four hours after plating, cells were selected in growth medium containing $200 \mu \mathrm{g} / \mathrm{mL}$ Hygromycin B with or without the addition of mitomycin $\mathrm{C}(4 \mathrm{ng} / \mathrm{mL})$. Colonies were harvested approximately twelve days after selection and fixed with $100 \%$ ice-cold methanol prior to staining with Giemsa. Colonies containing $\geq 50$ cells were scored positive. The percentage of mitomycin $\mathrm{C}$ resistant colonies was determined by dividing the number surviving selection with mitomycin $\mathrm{C}$ and Hygromycin $\mathrm{B}$ by the number that grew in the presence of Hygromycin $\mathrm{B}$ alone on the duplicate plate. Statistical significance of the experimental data was determined using SPSS ${ }^{\circledast}$ version 11.0 for Mac OS X. The mean numbers of percentage mitomycin $\mathrm{C}$ resistance for each construct were compared by ANOVA.

\section{Protein expression and western blotting}

Protein expression and Western blotting was conducted as described previously [32] with the exception that each sample was resolved on a 15\% SDS-PAGE. For detection of the smaller RAD51D $\Delta 3$ and RAD51D+int 3 constructs, cells harvested from two separate wells per sample were 
pooled and $200 \mu \mathrm{g}$ of each sample was resolved on 10$20 \%$ Tricine gel followed by transfer onto a 0.1 micron nitrocellulose membrane (Protran BA-79, Whattman).

\section{Expression analysis of Rad5 Id alternative splice variants}

Total RNA was purified from C57BL/6J mouse tissues (Aurum Total RNA Isolation kit; Bio-Rad) and reverse transcribed using both oligo(dT) and random hexamers (iScript cDNA Synthesis kit; Bio-Rad). Primers specific for Rad51d (Rad51d F1, Rad51d F2, Rad51d R1, and Rad51d R2), Rad51d 9,10 (Rad51d F3 and $\operatorname{Rad} 51 d \Delta 9,10 \mathrm{R} 1)$, or Rad51d 10 (Rad51d F7 and $\operatorname{Rad} 51 \mathrm{~d} \Delta 10$ R1) were employed. Amplification was performed under the following conditions: $94^{\circ} \mathrm{C}$ for $3 \mathrm{~min}$, followed by 35 cycles at $94^{\circ} \mathrm{C}$ for $30 \mathrm{~s}, 60^{\circ} \mathrm{C}$ for $30 \mathrm{~s}$, and $72^{\circ} \mathrm{C}$ for $1 \mathrm{~min}$. All sequences of primers used in this study are listed in Table 2. For expression analysis in human cells, total RNA from normal breast tissue (Stratagene, \#735044) was reverse transcribed as above and PCR amplified for 35 cycles using the HSTRF1 and HSTRR1 primers, corresponding to exon 2 and exon 6 respectively, as initially described [16]. The products were separated on a $5 \%$ polyacrylamide gel. Image J software http://rsbweb.nih.gov/ij/ was used for quantitative analysis of band intensity levels by the gel analysis function.

\section{Rapid amplification of Rad5 Id 3' cDNA ends}

Total RNA from pooled adult mouse BALB/c livers (Clontech) was used for 3'RACE according to manufacturer instructions (Gene Racer kit; Invitrogen). Amplification was performed using the GeneRacer 3' Primer and Rad51d F1. Nested PCR was carried out with the Gene Racer Nested Primer and Rad51d F2. Amplification products were cloned into pCR4-TOPO (Invitrogen), and sequences of newly identified isoforms deposited into GenBank [GenBank: EU627687 (RAD51D 49,10), EU627688 (RAD51D $\Delta 10)]$.

\section{Authors' contributions}

AMG and BDY equally contributed to the work presented in this manuscript. AMG and DLP conceived this study, and $A M G$ was responsible for generating the cDNA clones, complementation studies, $\mathrm{Y} 2 \mathrm{H}$ and localization analyses. BDY identified the new splice isoforms and performed the transcription studies. CR was responsible for verification of transgene expression. CM performed the molecular modeling. DLP participated as a supervisor in study design and analysis. He is responsible for manuscript drafts, along with AMG and BDY, and has given final approval for the version to be published.

Table 2: Primers used for cloning and expression studies

\begin{tabular}{|c|c|}
\hline Primer Name & Sequence \\
\hline Rad5Id FI & 5'-GCTGACTTGGAGGAAGTAGCCCAGAAGTGT-3' \\
\hline Rad5Id F2 & 5'-CTACTTGATGCTGGCCTCTATACTGG-3' \\
\hline Rad5Id F3 & 5'-GCAGGAAGCAACTTCTTCAGGCG-3' \\
\hline Rad5Id RI & 5'-AGCCTGTAGTAGCTGGAGGAGG-3' \\
\hline Rad5Id R2 & 5'-TGAACGCACCACCTGTATCCTCTGGAGAG-3' \\
\hline $\operatorname{Rad} 5 \mathrm{Id} \Delta 9,10 \mathrm{RI}$ & 5'-GTTCTAAGACAGACAGAGCAC-3' \\
\hline $\operatorname{Rad5Id} \Delta \mathrm{IO} \mathrm{RI}$ & 5'-GAGACACAGGTTCTTCACCACAC-3' \\
\hline $\operatorname{Rad} 5$ Id $\Delta 8$ Stul & 5'-TGACCAACCACTTGACTCGAGATTGGGATGGTAGG-3' \\
\hline $\operatorname{Rad} 5 \mathrm{Id} \Delta 8 \mathrm{BamHI}$ & 5'-GATCCCTACCATCCCAATCTCGAGTCAAGTGGTTGGTCA-3' \\
\hline $\operatorname{Rad5} I d \Delta 7 \mathrm{~b} F I$ & 5'-GCCCCACTTCTGGGAGGCCTGGCCTTGATG-3' \\
\hline $\operatorname{Rad5ID} \Delta 7 \mathrm{~b} R$ I & 5'-CATCAAGGCCAGGCCTCCCAGAAGTGGGGC-3' \\
\hline $\operatorname{Rad} 5$ Id $\Delta 7,8$ Avall & 5'-GACCTTCGCGGCACCATAGCCCAGCAGGTGACCAACCACTTGAC-3' \\
\hline $\operatorname{Rad} 5$ Id $\Delta 7,8$ Xhol & 5'-TGCAGTCAAGTGGTTGGTCACCTGCTGGGCTATGGTGCCGCGAAG-3' \\
\hline $\operatorname{Rad} 51 \mathrm{~d} \Delta 3$ BstXI & 5'-GTGGCTTGTCCTACAAGCCTGGACAAACTACTTGAG-3' \\
\hline Rad5IdKpnIF & 5'-GGACTATGGGTACCCTCAGGGCA-3' \\
\hline $\operatorname{Rad} 5$ Id $\Delta 5$ NheBamR & 5'-CAGGATCCAGCTAGCCTGGGTTTTGCCC-3' \\
\hline $\operatorname{Rad} 5 \mathrm{Id} \Delta 5 \mathrm{NheF}$ & 5'-AACTCAGGCTAGCGCTCTCCAGAGG-3' \\
\hline Rad5IdBamR & 5'-CAGTGGATCCCAATCAACAGTGTCA-3' \\
\hline $\operatorname{Rad} 5 \mathrm{Id}+\mathrm{int} 3 \mathrm{~F}$ & $\begin{array}{l}\text { 5'-CCGGCATCGGAAGGTTTGTATGCGAACCTGGACAAGCCTGGACAA } \\
\text { ACTACTTGATGCTGGCCTCTATACTGGGGAAGTGAG-3' }\end{array}$ \\
\hline Rad5Id+int3R & $\begin{array}{l}\text { 5'-GATCCCTCACTTCCCCAGTATAGAGGCCAGCATCAAGTAGTTTGTC } \\
\text { CAGGCTTGTCCAGGTTCGCATACAAACCTTCCGATG-3' }\end{array}$ \\
\hline Rad5Idex6F & 5'-CCAGAGGATACAGGTGGTGCGTTCATTTGAC-3' \\
\hline $\operatorname{Rad} 5 \mathrm{Id} \Delta 9,10 \mathrm{BamR}$ & 5'-CCGGGATCCGTTCTAAGACAGACAGAGCAC-3' \\
\hline $\operatorname{Rad} 5 \mathrm{Id} \Delta \mathrm{IOBamR}$ & 5'-CCGGGATCCGAGCACAGGTTCTTCACCA-3' \\
\hline Rad5IcKpnF & 5'-CTTGGTACCCAGCGGGAGTTGGTGGGT-3' \\
\hline Rad5 IcBamR & 5'-CAGTTAACTGGATCCACTGGCA-3' \\
\hline $\mathrm{XRCC} 2 \mathrm{~F}$ & 5'-ATGCTACGGCTCGTGACAGTTCTT-3' \\
\hline$X R C C 2 R$ & 5'-AGAAGATGACCCTGTGCTTCACGA-3' \\
\hline
\end{tabular}




\section{Additional material}

\section{Additional file 1}

Intracellular localization of RAD51D isoforms. (A) Localization of over-expressed EGFP-RAD51D isoforms. Panels represent images taken from cells transfected with the following DNA constructs: EGFP vector control (a), EGFP-RAD51C (b), EGFP-RAD51D-FL (c), EGFPRAD51D 8 (d), EGFP-RAD51D $7 b$ (e), EGFP-RAD51D 7,8 (f), EGFP-RAD51D $3(\mathrm{~g})$, EGFP-RAD51D $5(\mathrm{~h})$, EGFP-RAD51D+int3 $(i)$. (B) Repair activity of RAD51D tagged with amino-terminal enhanced green fluorescent protein. Rad51d-deficient mouse embryonic fibroblasts were challenged with $4 \mathrm{ng} / \mathrm{mL}$ mitomycin $C$ following transfection. Error bars represent the standard error. Abbreviations: 51D; RAD51D-FL (no tag), GFP; EGFP-RAD51D-FL, Vec; pcDNA3.1/Hygro vector.

Click here for file

[http://www.biomedcentral.com/content/supplementary/14712199-10-27-S1.jpeg]

\section{Acknowledgements}

We thank Joanna Albala and Kristi Miller for the kind gift of the Mus musculus Rad5 I d deletion constructs. This work was supported by an American Cancer Society grant (RSG-030I58-0I-GMC) to DLP.

\section{References}

I. Li X, Heyer WD: Homologous recombination in DNA repair and DNA damage tolerance. Cell Res 2008, 18:99-113.

2. Thacker J: The RAD5 I gene family, genetic instability and cancer. Cancer Lett 2005, 219:125-135.

3. West SC: Molecular views of recombination proteins and their control. Nat Rev Mol Cell Biol 2003, 4:435-445.

4. Takata M, Sasaki MS, Sonoda E, Fukushima T, Morrison C, Albala JS, Swagemakers SM, Kanaar R, Thompson LH, Takeda S: The Rad5 I paralog Rad5IB promotes homologous recombinational repair. Mol Cell Biol 2000, 20:6476-6482.

5. French CA, Masson JY, Griffin CS, O'Regan P, West SC, Thacker J: Role of mammalian RAD5 IL2 (RAD5 IC) in recombination and genetic stability. J Biol Chem 2002, 277:19322-19330.

6. Smiraldo PG, Gruver AM, Osborn JC, Pittman DL: Extensive chromosomal instability in Rad5 Id-deficient mouse cells. Cancer Res 2005, 65:2089-2096.

7. Liu N, Lamerdin JE, Tebbs RS, Schild D, Tucker JD, Shen MR, Brookman KW, Siciliano MJ, Walter CA, Fan W, Narayana LS, Zhou ZQ, Adamson AW, Sorensen KJ, Chen DJ, Jones NJ, Thompson LH: XRCC2 and XRCC3, new human Rad5I-family members, promote chromosome stability and protect against DNA cross-links and other damages. Mol Cell 1998, I:783-793.

8. Yoshida K, Kondoh G, Matsuda Y, Habu T, Nishimune Y, Morita T: The mouse RecA-like gene Dmcl is required for homologous chromosome synapsis during meiosis. Mol Cell 1998, I:707-718.

9. Masson JY, Tarsounas MC, Stasiak AZ, Stasiak A, Shah R, Mcllwraith $\mathrm{MJ}$, Benson FE, West SC: Identification and purification of two distinct complexes containing the five RAD5 I paralogs. Genes Dev 200I, I 5:3296-3307.

10. Wiese C, Collins DW, Albala JS, Thompson LH, Kronenberg A, Schild $D$ : Interactions involving the Rad5I paralogs Rad5IC and XRCC3 in human cells. Nucleic Acids Res 2002, 30: 100 I- 1008.

II. Tarsounas M, Munoz P, Claas A, Smiraldo PG, Pittman DL, Blasco MA, West SC: Telomere maintenance requires the RAD5ID recombination/repair protein. Cell 2004, I I 7:337-347.

12. Birzele F, Csaba G, Zimmer R: Alternative splicing and protein structure evolution. Nucleic Acids Res 2008, 36:550-558.

13. Ben-Dov C, Hartmann B, Lundgren J, Valcarcel J: Genome-wide analysis of alternative pre-mRNA splicing. J Biol Chem 2008, 283: $1229-1233$.
14. Romero PR, Zaidi S, Fang YY, Uversky VN, Radivojac P, Oldfield CJ, Cortese MS, Sickmeier M, LeGall T, Obradovic Z, Dunker AK: Alternative splicing in concert with protein intrinsic disorder enables increased functional diversity in multicellular organisms. Proc Natl Acad Sci USA 2006, 103:8390-8395.

15. Kawabata M, Akiyama K, Kawabata T: Genomic structure and multiple alternative transcripts of the mouse TRAD/ RAD5IL3/RAD5ID gene, a member of the recA/RAD5 I gene family. Biochim Biophys Acta 2004, 1679:107-116.

16. Kawabata M, Saeki K: Multiple alternative transcripts of the human homologue of the mouse TRAD/R5IH3/RAD5ID gene, a member of the rec A/RAD5 I gene family. Biochem Biophys Res Commun 1999, 257:156-162.

17. Pospisil H, Herrmann A, Bortfeldt RH, Reich JG: EASED: Extended Alternatively Spliced EST Database. Nucleic Acids Res 2004, 32:D70-4

18. Durrant WE, Wang S, Dong $X$ : Arabidopsis SNII and RAD5 ID regulate both gene transcription and DNA recombination during the defense response. Proc Natl Acad Sci USA 2007, 104:4223-4227.

19. Venables JP: Aberrant and alternative splicing in cancer. Cancer Res 2004, 64:7647-7654.

20. Srebrow A, Kornblihtt AR: The connection between splicing and cancer. J Cell Sci 2006, I 1 9:2635-264I.

21. Thorpe PH, Marrero VA, Savitzky MH, Sunjevaric I, Freeman TC, Rothstein R: Cells expressing murine RAD52 splice variants favor sister chromatid repair. Mol Cell Biol 2006, 26:3752-3763.

22. Farrugia DJ, Agarwal MK, Pankratz VS, Deffenbaugh AM, Pruss D, Frye C, Wadum L, Johnson K, Mentlick J, Tavtigian SV, Goldgar DE, Couch FJ: Functional assays for classification of BRCA2 variants of uncertain significance. Cancer Res 2008, 68:3523-3531.

23. Claes K, Poppe B, Machackova E, Coene I, Foretova L, De Paepe A, Messiaen L: Differentiating pathogenic mutations from polymorphic alterations in the splice sites of BRCAI and BRCA2. Genes Chromosomes Cancer 2003, 37:3 I4-320.

24. Park JY, Yoo HW, Kim BR, Park R, Choi SY, Kim Y: Identification of a novel human Rad5 I variant that promotes DNA strand exchange. Nucleic Acids Res 2008, 36:3226-3234.

25. Pittman DL, Weinberg LR, Schimenti JC: Identification, characterization, and genetic mapping of Rad5 Id, a new mouse and human RAD5 I/RecA-related gene. Genomics 1998, 49: I03-III.

26. Thanaraj TA, Stamm S, Clark F, Riethoven JJ, Le Texier V, Muilu J: ASD: the Alternative Splicing Database. Nucleic Acids Res 2004, 32:D64-9.

27. Schild D, Lio YC, Collins DW, Tsomondo T, Chen DJ: Evidence for simultaneous protein interactions between human Rad5 I paralogs. J Biol Chem 2000, 275: I6443-16449.

28. Dosanjh MK, Collins DW, Fan W, Lennon GG, Albala JS, Shen Z, Schild D: Isolation and characterization of RAD5 IC, a new human member of the RAD5 I family of related genes. Nucleic Acids Res 1998, 26: I 179-1 I84.

29. Miller KA, Sawicka D, Barsky D, Albala JS: Domain mapping of the Rad5I paralog protein complexes. Nucleic Acids Res 2004, 32:169-178.

30. French CA, Tambini CE, Thacker J: Identification of functional domains in the RAD5 IL2 (RAD5 IC) protein and its requirement for gene conversion. J Biol Chem 2003, 278:45445-45450.

31. Miller KA, Hinz JM, Yamada NA, Thompson LH, Albala JS: Nuclear localization of $\operatorname{Rad5IB}$ is independent of $\operatorname{Rad5IC}$ and BRCA2. Mutagenesis 2005, 20:57-63.

32. Gruver AM, Miller KA, Rajesh C, Smiraldo PG, Kaliyaperumal S, Balder R, Stiles KM, Albala JS, Pittman DL: The ATPase motif in RAD5ID is required for resistance to DNA interstrand crosslinking agents and interaction with RAD5 IC. Mutagenesis 2005, 20:433-440.

33. Yokoyama H, Sarai N, Kagawa W, Enomoto R, Shibata T, Kurumizaka $H$, Yokoyama S: Preferential binding to branched DNA strands and strand-annealing activity of the human Rad5 IB, Rad5 IC, Rad5ID and Xrcc2 protein complex. Nucleic Acids Res 2004, 32:2556-2565.

34. Rodriguez-Lopez R, Osorio A, Ribas G, Pollan M, Sanchez-Pulido L, de la Hoya M, Ruibal A, Zamora P, Arias Jl, Salazar R, Vega A, Martinez II, Esteban-Cardenosa E, Alonso C, Leton R, Urioste Azcorra M, Miner C, Armengod ME, Carracedo A, Gonzalez-Sarmiento R, Caldes 
T, Diez O, Benitez J: The variant E233G of the RAD5 ID gene could be a low-penetrance allele in high-risk breast cancer families without BRCAI/2 mutations. Int J Cancer 2004, I I 0:845-849.

35. Kurumizaka H, lkawa S, Nakada M, Enomoto R, Kagawa W, Kinebuchi $\mathrm{T}$, Yamazoe M, Yokoyama S, Shibata T: Homologous pairing and ring and filament structure formation activities of the human Xrcc2*Rad5ID complex. J Biol Chem 2002, 277:14315-14320.

36. Pittman DL, Schimenti JC: Midgestation lethality in mice deficient for the RecA-related gene, Rad5Id/Rad5II3. Genesis 2000, 26:167-173

37. Wiese C, Hinz JM, Tebbs RS, Nham PB, Urbin SS, Collins DW, Thompson LH, Schild D: Disparate requirements for the Walker A and B ATPase motifs of human RAD5ID in homologous recombination. Nucleic Acids Res 2006, 34:2833-2843.

38. Orelle C, Dalmas O, Gros P, Di Pietro A, Jault JM: The conserved glutamate residue adjacent to the Walker-B motif is the catalytic base for ATP hydrolysis in the ATP-binding cassette transporter BmrA. J Biol Chem 2003, 278:47002-47008.

39. Schoenmakers EF, Huysmans C, Ven WJ Van de: Allelic knockout of novel splice variants of human recombination repair gene RAD5 I B in $\mathbf{t}(\mathbf{1} \mathbf{2} ; \mathbf{1 4})$ uterine leiomyomas. Cancer Res 1999, 59:19-23.

40. Connell PP, Siddiqui N, Hoffman S, Kuang A, Khatipov EA, Weichselbaum RR, Bishop DK: A hot spot for RAD5IC interactions revealed by a peptide that sensitizes cells to cisplatin. Cancer Res 2004, 64:3002-3005.

4I. Sampath J, Pelus LM: Alternative splice variants of survivin as potential targets in cancer. Curr Drug Discov Technol 2007, 4:174-191.

42. Gaur RK: RNA interference: a potential therapeutic tool for silencing splice isoforms linked to human diseases. Biotechniques 2006:15-22.

43. Chang YF, Imam JS, Wilkinson MF: The nonsense-mediated decay RNA surveillance pathway. Annu Rev Biochem 2007, 76:5I-74.

44. Lewis BP, Green RE, Brenner SE: Evidence for the widespread coupling of alternative splicing and nonsense-mediated mRNA decay in humans. Proc Natl Acad Sci USA 2003, 100:189-192.

45. Lareau LF, Brooks AN, Soergel DA, Meng Q, Brenner SE: The coupling of alternative splicing and nonsense-mediated mRNA decay. Adv Exp Med Biol 2007, 623:190-21 I

46. Lejeune F, Maquat LE: Mechanistic links between nonsensemediated mRNA decay and pre-mRNA splicing in mammalian cells. Curr Opin Cell Biol 2005, I 7:309-3I5.

47. Braybrooke JP, Li JL, Wu L, Caple F, Benson FE, Hickson ID: Functional interaction between the Bloom's syndrome helicase and the RAD5 I paralog, RAD5IL3 (RAD5ID). J Biol Chem 2003, 278:48357-48366.

48. Martin V, Chahwan C, Gao H, Blais V, Wohlschlegel J, Yates JRr, McGowan $\mathrm{CH}$, Russell $\mathrm{P}$ : Sws I is a conserved regulator of homologous recombination in eukaryotic cells. EMBO J 2006, 25:2564-2574
Publish with Biomed Central and every scientist can read your work free of charge

"BioMed Central will be the most significant development for disseminating the results of biomedical research in our lifetime. "

Sir Paul Nurse, Cancer Research UK

Your research papers will be:

- available free of charge to the entire biomedical community

- peer reviewed and published immediately upon acceptance

- cited in PubMed and archived on PubMed Central

- yours - you keep the copyright
BioMedcentral 